\title{
Some aspects on the correlation between the kappa-symmetric and the pure spinor versions of the supermembrane in $D=11$
}

\author{
Elena-Mirela Băbălîc* \\ IFIN-HH, Department of Theoretical Physics, Str. Atomistilor no.407, Bucharest-Magurele, \\ ROMANIA \\ E-mail: mbabalicetheory.nipne.ro
}

\begin{abstract}
We studied a relation between the $\kappa$-symmetric and the pure spinor formulations of the supermembrane in eleven dimensions using Berkovits' method for the superstring in $D=10$. Here we attempt to extend this method to the supermembrane showing that it is possible to reinstate the reparameterisation constraints in the pure-spinor formulation of the supermembrane if we introduce a topological sector and performe a similarity transformation. The resulting BRST charge is then of conventional type and is argued to be (related to) the BRST charge of the $\kappa$-symmetric supermembrane in a formulation where all second class constraints are 'gauge unfixed' to first class constraints. In this analysis we also encounter a natural candidate for a (non-covariant) supermembrane analogue of the superstring $b$ ghost.
\end{abstract}

Proceedings of the Corfu Summer Institute 2011 School and Workshops on Elementary Particle Physics and Gravity

September 4-18, 2011

Corfu, Greece

\footnotetext{
* Speaker.
} 


\section{Introduction}

There are two essential problems that appear when trying to quantize the supermembrane: the first, also present for the bosonic supermembrane, is related to the complicated, more than quadratic nature of the Hamiltonian, which leads to difficulties in constructing the physical spectrum, and the second, also present for the Green-Schwartz superstring, refers to the presence of some fermionic constraints due to the kappa-symmetry of the action, constraints that can not be separated in first and second class in a Lorentz-covariant manner. They have been separated only with the price of breaking the covariance by using light-cone coordinates.

Around ten years ago, a new approach started to be used, mostly by Berkovits, for the superstring - the pure spinor formalism [2], which has proven to be quite useful for quantising the superstring in a manifestly super-Poincaré covariant manner. The Green-Schwarz formulation of the open superstring (or one of the two sectors of the closed superstring) has one reparameterisation constraint, $T$, and 16 fermionic constraints, $d_{\alpha}$, half of which are second class and the other half first class. The separation of the two types of constraints in a Lorentz-covariant manner, preserving the full ten-dimensional symmetry, is not possible. Giving up manifest covariance,the usual way to treat the $d_{\alpha}$ constraints starts by constructing the Dirac bracket from the second class constraints. Instead of this approach, an alternative is to try to view the eight second class constraints as four first class constraints plus four gauge fixing conditions. In the case of the superstring the resulting set of twelve fermionic first class constraints can then, together with $T$, be used to write a conventional BRST charge. This BRST charge is not manifestly Lorentz covariant. However, after a similarity transformation it can be shown to be equal to the pure spinor BRST charge plus a topological term which decouples due to the quartet mechanism [2].

After the decoupling, the resulting BRST charge agrees with the pure spinor BRST charge thereby establishing the equivalence between the two formalisms. The decoupling of the topological quartet effectively removes the reparameterisation constraint together with one of the fermionic constraints and the corresponding ghosts, and reinstates Lorentz covariance. The remaining eleven bosonic ghosts build up a pure spinor - eleven being the dimension of such a spinor in ten dimensions.

So, in this new approach, the starting point is an action in which the gauge kappa-symmetry is replaced by a rigid symmetry of the BRST type, whose generator $Q$ called "BRST charge" is chosen linear in the fermionic constraints of the kappa-symmetric formulation and the components of a bosonic ghost which satisfies the pure spinor condition. It was shown that physical states for the superstring belong to the cohomology of $Q$. After adding some non-minimal fields and performing a similarity transformation the "BRST charge" $Q$ in the pure spinor formalism gets a conventional form argued to be equivalent to the one in the kappa-symmetric formalism.

The present paper is an attempt to extend this pure spinor method to the case of the supermembrane in eleven dimensions, in the sense of finding a connection between the "BRST charge" postulated in the pure spinor aproach and the conventional BRST charge in the kappa-symmetric version. It is based almost entirely on the results we obtained in [1]. 


\section{Supermembrane in eleven dimensions}

In this section we discuss the extension of the method described in the introduction to the supermembrane. We take as a toy model the superparticle case in eleven dimensions studied in [1].

\subsection{The $\kappa$-symmetric supermembrane}

The starting point is the BST (Bergshoeff-Sezggin-Townsend) [4] action for the supermembrane in $\mathrm{D}=11$ in a flat supergravity background

$$
\begin{aligned}
S= & \int \mathrm{d} \tau \mathrm{d}^{2} \sigma\left[P_{M} \Pi_{0}^{M}+e^{0}\left(P_{M} P^{M}+\Delta\right)+e^{i} \Pi_{i}^{M} P_{M}\right. \\
& \left.-\frac{i}{2} \varepsilon^{I J K}\left(\theta \Gamma_{M N} \partial_{I} \theta\right)\left[\Pi_{J}^{M} \Pi_{K}^{N}+i \Pi_{J}^{M}\left(\theta \Gamma^{N} \partial_{K} \theta\right)-\frac{1}{3}\left(\theta \Gamma^{M} \partial_{J} \theta\right)\left(\theta \Gamma^{N} \partial_{K} \theta\right)\right]\right] \\
= & -\frac{1}{2} \int \mathrm{d}^{3} \zeta\left[\sqrt{-g}\left(g^{I J} \Pi_{I}^{M} \Pi_{J M}-1\right)\right. \\
& \left.+i \varepsilon^{I J K}\left(\theta \Gamma_{M N} \partial_{I} \theta\right)\left[\Pi_{J}^{M} \Pi_{K}^{N}+i \Pi_{J}^{M}\left(\theta \Gamma^{N} \partial_{K} \theta\right)-\frac{1}{3}\left(\theta \Gamma^{M} \partial_{J} \theta\right)\left(\theta \Gamma^{N} \partial_{K} \theta\right)\right]\right],
\end{aligned}
$$

which is invariant under global supersymmetry as well as under a local (gauge) fermionic symmetry known as the $\kappa$-symmetry [8].

$\zeta^{I}=\left(\tau, \sigma^{i}\right)$ are the space-time coordinates on the worldvolume, $I, J, K=0,1,2$ and $i, j=1,2$, while $e^{0}$ and $e^{i}$ are the components of a vielbein.

We also used the folowing notations

$$
\begin{gathered}
\Pi_{I}^{M}=\partial_{I} X^{M}-i \theta \Gamma^{M} \partial_{I} \theta, \\
\Delta=\operatorname{det}\left(\Pi_{i}^{N} \Pi_{j N}\right)=\frac{1}{2} \varepsilon^{i j} \Pi_{i}^{M} \Pi_{j}^{N} \varepsilon^{k l} \Pi_{k M} \Pi_{l N},
\end{gathered}
$$

where $\Pi_{I}^{M}\left(\tau, \sigma^{i}\right)$ are the components of the supersymmetric momentum, $X^{M}\left(\tau, \sigma^{i}\right)$ the bosonic coordinates for the supermembrane in superspace whith associated conjugate momenta $P_{M}\left(\tau, \sigma^{i}\right)$, the fermionic objects $\theta^{A}\left(\tau, \sigma^{i}\right)$ are the components of a Majorana spinor and have $p_{A}\left(\tau, \sigma^{i}\right)$ as their associated conjugate momenta. $\left(\Gamma^{M}\right)_{A B}$ are hermitic and symmetric matrices in Majorana representasion. We denoted Lorentz indices by $M=0, \ldots, 9,11$ and spinor indices by $A=1, \ldots, 32$. For more conventions and technical details see appendix A.

The two forms of the action above are related by integrating out $P_{M}$ and using the parameterisation $g_{i j} \rightarrow\left(\gamma_{i j}, N, N_{i}\right)[7]$

$$
\begin{gathered}
g_{i j}=\gamma_{i j}, \quad g_{0 i}=2 \gamma_{i j} N^{j}, \quad g_{00}=-N^{2}+\gamma_{i j} N^{i} N^{j}, \\
g^{i j}=\gamma^{i j}-\frac{N^{i} N^{j}}{N^{2}}, \quad g^{0 i}=\frac{N^{i}}{N^{2}}, \quad g^{00}=-\frac{1}{N^{2}}, \\
g=N \sqrt{\gamma}=N \sqrt{\operatorname{det} \gamma_{i j}},
\end{gathered}
$$

together with the identifications

$$
e^{0}=\frac{N}{2 \sqrt{\gamma}}, \quad e^{i}=-N^{i}
$$


and the result [7]

$$
\Delta=\gamma\left(\gamma^{i j} \Pi_{i}^{M} \Pi_{j M}-1\right)
$$

Of particular interest for us is the hamiltonian analysis of the constraints derived from the above action. The theory is subject to 32 fermionic primary constraints

$$
\begin{aligned}
d_{A}= & p_{A}-i P_{M}\left(\Gamma^{M} \theta\right)_{A} \\
& -\frac{i}{2} \varepsilon^{i j}\left(\Gamma_{M N} \theta\right)_{A}\left[\Pi_{i}^{M} \Pi_{j}^{N}+i \Pi_{i}^{M}\left(\theta \Gamma^{N} \partial_{j} \theta\right)-\frac{1}{3}\left(\theta \Gamma^{M} \partial_{i} \theta\right)\left(\theta \Gamma^{N} \partial_{j} \theta\right)\right] \\
& -\frac{1}{2} \varepsilon^{i j}\left(\theta \Gamma_{M N} \partial_{i} \theta\right)\left(\Gamma^{M} \theta\right)_{A}\left[\Pi_{j}^{N}+\frac{2 i}{3} \theta \Gamma^{N} \partial_{j} \theta\right] \approx 0 .
\end{aligned}
$$

and to 3 bosonic secondary constraints, known as the reparametrisation constraints

$$
\begin{aligned}
& T=K_{M} K^{M}+\Delta-2 \varepsilon^{i j} \Pi_{i}^{M}\left(d \Gamma_{M} \partial_{j} \theta\right) \approx 0, \\
& T_{i}=K_{M} \Pi_{i}^{M}-d \partial_{i} \theta \approx 0
\end{aligned}
$$

where we used notation

$$
K_{M}=P_{M}-i \varepsilon^{i j}\left(\theta \Gamma_{M N} \partial_{i} \theta\right)\left(\Pi_{j}^{N}+\frac{i}{2} \theta \Gamma^{N} \partial_{j} \theta\right)
$$

The basic canonical Poisson brackets are

$$
\left\{P_{M}(\sigma), X^{N}(\rho)\right\}=-\delta_{M}^{N} \delta^{2}(\sigma-\rho), \quad\left\{p_{A}(\sigma), \theta^{B}(\rho)\right\}=-\delta_{A}^{B} \delta^{2}(\sigma-\rho),
$$

which imply the following non-vanishing Poisson brackets between $K_{M}, \Pi_{i}^{M}$ and $d_{A}$ :

$$
\begin{aligned}
\left\{d_{A}(\sigma), d_{B}(\rho)\right\} & =2 i K_{M} \Gamma_{A B}^{M} \delta^{2}(\sigma-\rho)+i \varepsilon^{i j} \Pi_{i M} \Pi_{j N} \Gamma_{A B}^{M N} \delta^{2}(\sigma-\rho), \\
\left\{d_{A}(\sigma), K_{M}(\rho)\right\} & =-2 i \varepsilon^{i j} \Pi_{i}^{N}\left(\Gamma_{M N} \partial_{j} \theta\right)_{A} \delta^{2}(\sigma-\rho), \\
\left\{d_{A}(\sigma), \Pi_{i}^{M}(\rho)\right\} & =2 i\left(\Gamma^{M} \partial_{i} \theta\right)_{A} \delta^{2}(\sigma-\rho), \\
\left\{K_{M}(\sigma), K_{N}(\rho)\right\} & =-i \varepsilon^{i j}\left(\partial_{i} \theta \Gamma_{M N} \partial_{j} \theta\right) \delta^{2}(\sigma-\rho), \\
\left\{K_{M}(\sigma), \Pi_{i}^{N}(\rho)\right\} & =-\delta_{M}^{N} \frac{\partial}{\partial \rho_{i}} \delta^{2}(\sigma-\rho),
\end{aligned}
$$

where, all fields on the right hand side depend on $\rho$.

$T, T_{i}$ and half of $d_{A}$ are first class constraints, while the other half of $d_{A}$ are second class constraints. Because there is no way (at least not a simple known one) of covariantly separating the fermionic constraints, we can drop the covariance and work in light-cone coordinates. After a procedure of gauge un-fixing the second class constraints to first class constraints we can start writing down the BRST charge for this theory. But for a manifest Lorentz covariant description of the supermembrane we argue that the pure spinor method can be used. 


\subsection{The pure spinor supermembrane}

A pure spinor version of the supermembrane in eleven dimensions was proposed by Berkovits [5]. This model is based on the action

$$
\begin{aligned}
S= & \int \mathrm{d} \tau \mathrm{d}^{2} \sigma\left[K_{M} \Pi_{0}^{M}-d \partial_{0} \theta+w \partial_{0} \lambda\right. \\
& -\frac{i}{2} \varepsilon^{I J K}\left(\theta \Gamma_{M N} \partial_{I} \theta\right)\left(\Pi_{J}^{M} \Pi_{K}^{N}+i \Pi_{J}^{M}\left(\theta \Gamma^{N} \partial_{K} \theta\right)-\frac{1}{3}\left(\theta \Gamma^{M} \partial_{J} \theta\right)\left(\theta \Gamma^{N} \partial_{K} \theta\right)\right. \\
& -\frac{1}{2}\left[K_{M} K^{M}+M+2 \varepsilon^{i j}\left(d \Gamma_{M} \partial_{i} \theta\right) \Pi_{j}^{M}+2 \varepsilon^{i j}\left(w \Gamma_{M} \partial_{i} \lambda\right) \Pi_{j}^{M}\right. \\
& \left.+4 i \varepsilon^{i j}\left(w \Gamma^{M} \partial_{i} \theta\right)\left(\lambda \Gamma_{M} \partial_{j} \theta\right)-4 i \varepsilon^{i j}\left(w \partial_{i} \theta\right)\left(\lambda \partial_{j} \theta\right)\right] \\
& \left.+e^{i}\left[K_{M} \Pi_{i}^{M}-d \partial_{i} \theta+w \partial_{i} \lambda\right]\right],
\end{aligned}
$$

where new variables appear: a bosonic ghost $\left\{\lambda^{A}\right\}_{A=1 \ldots 32}$, which is a pure spinor (i.e. it satisfies a pure spinor constraint) and its conjugate momentum $\left\{w_{A}\right\}_{A=1 \ldots 32}$. The pure spinor constraint

$$
\lambda \Gamma^{M} \lambda=0
$$

leaves $\lambda^{A}$ with only 23 independent components and induces a gauge invariance

$$
\delta w_{A}=\Lambda_{M}\left(\Gamma^{M} \lambda\right)_{A},
$$

which means that $w_{A}$ and $\lambda^{A}$ do not have a canonical (Lorentz covariant) Poisson bracket. However, from $w$ and $\lambda$ one can form gauge-invariant Lorentz-covariant objects, such as

$$
J=w \lambda, \quad N^{M N}=\frac{1}{2}\left(w \Gamma^{M N} \lambda\right)
$$

which correspond to the ghost charge and respectively the Lorentz current in the $(w, \lambda)$ sector. For calculations involving such gauge invariant expressions, one can effectively use the canonical Poisson bracket $\left\{w_{A}, \lambda^{B}\right\}=-\delta_{A}^{B}$, as the non-covariant pieces cancel.

The proposed BRST charge is

$$
Q=\int \mathrm{d}^{2} \sigma \lambda^{A} d_{A}
$$

However, in contrast to the superparticle case studied in [1], further constraints are needed to make $Q$ nilpotent and the action BRST invariant. As shown in [5] the following constraints also seem to be required

$$
\left(\lambda \Gamma_{M N} \lambda\right) \Pi_{i}^{N}=0, \quad \lambda \partial_{i} \lambda=0 .
$$

Note that the constraints (2.16) are BRST closed. Still, the exact form of the full set of constraints deserves further study.

We should also mention another attempt to understand the origin of the pure spinor supermembrane [8]. In this paper the goal was to derive the pure spinor model starting from a "doubled" version of the $\kappa$-symmetric supermembrane. This approach was partially successful, but was not as complete as that for the superstring [9]. 


\subsection{Relation between the two formulations}

In order to discuss the relation between the above two formulations we restrict ourselves to a classical analysis (i.e. work at the level of Poisson brackets). Our discussion closely parallels the ten-dimensional case discussed in [3].

A natural first step is to try to find a supermembrane generalisation of the superparticle result in [1]. The first step is to introduce a topological quartet $(b, c, \beta, \gamma)$ to the pure spinor BRST charge which ads to $Q$ a cohomological trivial term

$$
Q \rightarrow Q^{\prime}=\lambda^{A} d_{A}+b \gamma
$$

Here $b, c$ are fermionic and $\beta, \gamma$ are bosonic. The canonical Poisson brackets between the new variables are

$$
\{b, c\}=-1, \quad\{\beta, \gamma\}=-1 .
$$

Then, one performs the similarity transformation

$$
Q^{\prime \prime}=e^{c R / \gamma} Q^{\prime} e^{-c R / \gamma} \equiv Q^{\prime}+\left\{\frac{c R}{\gamma}, Q^{\prime}\right\}+\frac{1}{2 !}\left\{\frac{c R}{\gamma},\left\{\frac{c R}{\gamma}, Q^{\prime}\right\}\right\}+\ldots,
$$

where we propose the following expression for $R$ in order to provide a suitable generalisation to the supermembrane case

$$
\begin{aligned}
R= & \int \mathrm{d}^{2} \sigma\left[-\frac{i}{2} K_{M}\left(d \Gamma^{M} \xi\right)+\frac{i}{4} \varepsilon^{i j} \Pi_{i}^{M} \Pi_{j}^{N}\left(d \Gamma_{M N} \xi\right)\right. \\
& -\frac{1}{2} \varepsilon^{i j} \Pi_{i}^{M}\left(\xi \Gamma_{M} \partial_{j} \theta\right)(w \lambda)-\frac{1}{4} \varepsilon^{i j} \Pi_{i}^{M}\left(\xi \Gamma_{M N R} \partial_{j} \theta\right)\left(w \Gamma^{N R} \lambda\right) \\
& \left.-\frac{1}{2} \varepsilon^{i j} \Pi_{i}^{M}\left(\xi \partial_{j} \theta\right)\left(w \Gamma_{M} \lambda\right)-\frac{1}{4} \varepsilon^{i j} \Pi_{i}^{M}\left(\xi \Gamma^{N R} \partial_{j} \theta\right)\left(w \Gamma_{M N R} \lambda\right)\right] .
\end{aligned}
$$

A few comments about this expression are in order. The second line of this expression is invariant under the gauge transformation $\delta w_{A}=\Lambda_{M}\left(\Gamma^{M} \lambda\right)_{A}$ arising from the fact that $\lambda$ is pure, i.e. $\lambda \Gamma^{M} \lambda=$ 0 . This is easy to see since it involves the gauge invariant expressions encountered previously in the superparticle case (2.14). The first term on the third line is not invariant unless one imposes additional conditions. Provided that $\left(\lambda \Gamma_{M N} \lambda\right) \Pi_{i}^{M}=0$ it is invariant. However, even with this additional condition the second term on the third line of (2.20) is not invariant; instead its variation becomes proportional to $\Lambda_{P} \Pi_{i}^{P} \varepsilon^{i j}\left(\xi \Gamma_{M N} \partial_{j} \theta\right)\left(\lambda \Gamma^{M N} \lambda\right)$. If one imposes the stronger condition $\lambda \Gamma^{M N} \lambda=0$ (or the slightly weaker condition $\left(\lambda \Gamma^{M N} \lambda\right) \Pi_{i}^{P}=0$ ), it is invariant, but this possibility appears disfavoured since in previous work [5] constraints stronger than (2.16) were not necessary. Another possibility is that $\{Q, \delta R\}=0$, i.e. $R$ is only gauge invariant up to BRST closed terms. Although some terms in $\{Q, \delta R\}$ can be cancelled if one also imposes $\lambda \partial_{i} \lambda=0$, it seems that not all terms can be made to vanish, even using Fierz identities. Therefore we are left with a puzzle regarding the final term in (2.20). For the remainder of this paper we will assume that either the stronger condition $\lambda \Gamma^{M N} \lambda=0$ can be used in our calculations, or that there is another way to make the final term in (2.20) gauge invariant so that the non-covariant pieces in the bracket with $Q$ vanish.

A strong argument in favour of (2.20) is related to its behaviour under the double dimensional reduction to the $d=10$ type IIA superstring case. Under this reduction one has

$$
\Pi_{2}^{M}=\delta_{11}^{M}, \quad \partial_{2} \theta=0, \quad K_{11}=\Lambda_{11}=0 .
$$


It is easy to check that when these conditions are fulfilled, $R$ as written above is gauge invariant. Furthermore, by implementing the conditions (2.21) into $R$ leads to

$$
\begin{aligned}
R_{d=10}= & \int \mathrm{d} \sigma_{1}\left[-\frac{i}{2} K_{m}\left(d \Gamma^{m} \xi\right)+\frac{i}{2} \Pi_{1}^{m}\left(d \Gamma_{m} \Gamma^{11} \xi\right)\right. \\
& -\frac{1}{2}\left(\xi \Gamma^{11} \partial_{1} \theta\right)(w \lambda)-\frac{1}{4}\left(\xi \Gamma^{11} \Gamma_{n r} \partial_{1} \theta\right)\left(w \Gamma^{n r} \lambda\right) \\
& \left.-\frac{1}{2}\left(\xi \partial_{1} \theta\right)\left(w \Gamma^{11} \lambda\right)-\frac{1}{4}\left(\xi \Gamma_{n r} \partial_{1} \theta\right)\left(w \Gamma^{11} \Gamma^{n r} \lambda\right)\right]
\end{aligned}
$$

where $m, n, r=0, \ldots, 9$. Splitting $\xi^{A}=\left(\xi^{\alpha}, \tilde{\xi}^{\dot{\alpha}}\right)$ and similarly for $d_{A}, \theta_{A}, w_{A}$ and $\lambda^{A}$, we find $R=\xi^{\alpha} G_{\alpha}+\tilde{\xi}^{\dot{\alpha}} \tilde{G}_{\dot{\alpha}}$ where

$$
\begin{aligned}
G_{\alpha}= & -\frac{i}{2} K_{m}\left(d \gamma^{m}\right)_{\alpha}+\frac{i}{2} \Pi^{m}\left(d \gamma_{m}\right)_{\alpha} \\
& -\frac{1}{2}(\partial \theta)_{\alpha}(w \lambda)-\frac{1}{4}\left(\gamma_{m n} \partial \theta\right)_{\alpha}\left(w \gamma^{m n} \lambda\right), \\
\tilde{G}_{\dot{\alpha}}= & -\frac{i}{2} K_{m}\left(\tilde{d} \gamma^{m}\right)_{\dot{\alpha}}-\frac{i}{2} \Pi^{m}\left(\tilde{d} \gamma_{m}\right)_{\dot{\alpha}} \\
& -\frac{1}{2}(\bar{\partial} \tilde{\theta})_{\dot{\alpha}}(\tilde{w} \tilde{\lambda})-\frac{1}{4}\left(\gamma_{m n} \bar{\partial} \tilde{\theta}\right)_{\dot{\alpha}}\left(\tilde{w} \gamma^{m n} \tilde{\lambda}\right),
\end{aligned}
$$

which precisely corresponds to the ten-dimensional result [10].

$>$ From (2.20) a lengthy calculation leads to

$$
\{Q, R\}=\int \mathrm{d}^{2} \sigma\left[(\lambda \xi) \mathscr{T}-2\left(\lambda \Gamma_{M} \xi\right) \varepsilon^{i j} \Pi_{i}^{M} \mathscr{T}_{j}\right],
$$

where

$$
\begin{aligned}
\mathscr{T}= & K^{M} K_{M}+\Delta-2 \varepsilon^{i j} \Pi_{i}^{M}\left(d \Gamma_{M} \partial_{j} \theta\right)-2 \varepsilon^{i j} \Pi_{i}^{M}\left(w \Gamma_{M} \partial_{j} \lambda\right) \\
& -4 i \varepsilon^{i j}\left(w \partial_{i} \theta\right)\left(\lambda \partial_{j} \theta\right)+4 i \varepsilon^{i j}\left(w \Gamma_{M} \partial_{i} \theta\right)\left(\lambda \Gamma^{M} \partial_{j} \theta\right) \\
\mathscr{T}_{i}= & K_{M} \Pi_{i}^{M}-d \partial_{i} \theta+w \partial_{i} \lambda .
\end{aligned}
$$

This is one of the main results of this paper. Note that $\mathscr{T}$ and $\mathscr{T}_{i}$ in (2.25) are ghost completions of $T$ and $T_{i}$ in (2.7).

The expressions in (2.25) are precisely the combinations that appear in the third, the fourth, and the fifth lines of the action (2.11), which would look something like

$$
S=\int \mathrm{d} \tau \mathrm{d}^{2} \sigma\left[\ldots+\frac{1}{2} \mathscr{T}+e^{i} \mathscr{T} i\right]
$$

This is a good indication that we are on the right track, and gives further support to our proposal for $R$.

If one imposes $\lambda \Gamma_{M} \xi=0$ and $\lambda \xi=1$, one finds

$$
\{Q, R\}=T \text {. }
$$

This implies that $R$ is an eleven-dimensional analogue of the (non-covariant) superstring $b$ ghost (the superparticle limit of which was discussed in [6].) It is non-covariant in the sense of the $Y$-formalism [10]. The $b$ ghost appears in the calculation of the multiloop scattering amplitudes 
for the superstring and we assume that this $R$ found in the supermembrane case may play a similar role.

After performing a similarity transformation in first order we obtain a first term in the new "BRST charge" which depends on one of the reparametrisation constraints (starting to look like conventional form for a BRST charge)

$$
Q^{\prime \prime}=\int d^{2} \sigma(c T+\ldots)
$$

A natural strategy would be to also try to find an $R_{i}$ such that $\left\{Q, R_{i}\right\}=T_{i}+\ldots$. An attempt based on $R_{i}=\int \mathrm{d}^{2} \sigma \Pi_{i}^{M}\left(d \Gamma_{M} \xi\right)+\ldots$ fails since one is forced to impose $\lambda \Gamma^{M} \xi=0=\lambda \Gamma^{M N} \xi$ and $\lambda \xi \neq 0$. However, when $\left(\lambda \Gamma^{M} \lambda\right)=0$,

$$
(\lambda \xi)^{2}=\frac{3}{2}\left(\lambda \Gamma^{M} \xi\right)\left(\lambda \Gamma_{M} \xi\right)+\frac{1}{4}\left(\lambda \Gamma^{M N} \xi\right)\left(\lambda \Gamma_{M N} \xi\right) .
$$

As in the superparticle case one can perform transformations using $e^{c_{\xi} R_{\xi} / \gamma_{\xi}}$ where the $\xi$ subscript indicate that we perform several transformations using $R$ 's with various different fixed $\xi$ 's. This gives leading terms in $Q^{\prime}$ of the form

$$
Q^{\prime}=\int d^{2} \sigma\left(\sum_{\xi} c_{\xi} T_{\xi}+\ldots\right),
$$

where $T_{\xi}$ is a certain combination of $T$ and the eleven $T^{M} \equiv \varepsilon^{i j} \Pi_{i}^{M} T_{j}$.

Possibly the most natural approach would be to pick $T$ and two fixed $M$ 's, \pm say, $T^{ \pm}=$ $\varepsilon^{i j} \Pi_{i}^{ \pm} T_{j}$, so that

$$
Q^{\prime}=\int d^{2} \sigma\left(c T+c_{+} T^{+}+c_{-} T^{-}+\ldots\right)
$$

Although not covariant and not based on the usual form of reparameterisation constraints (2.7) this would be part of a viable form for a BRST charge arising from the $\kappa$-symmetric formulation. If one insists on covariance one could keep all the $T^{M}$ so that

$$
Q^{\prime}=\int d^{2} \sigma\left(c T+c_{M} T^{M}+\ldots\right) .
$$

In this case the constraints would be reducible, but it may be profitable to keep covariance i.e. to work with $T^{M}=\varepsilon^{i j} \Pi_{i}^{M} T_{j}$. It is easy to check that, generically, the two sets of constraints based on $T^{M}$ or $T_{i}$ define the same constraint surface.

Thus, we seem to be close to finding a $Q^{\prime}$ which can be related to a BRST charge in the $\kappa$ symmetric formulation, either of the form (2.31) or (2.32). This point should be studied further. Also, we only calculated the lowest order terms in the similarity transformation. Although general theorems seem to guarantee that the construction will work also at higher orders since we started from a BRST charge that satisfies $\{Q, Q\}=0$, it may be profitable to work out the details.

Above we only studied how the BRST charges are related. In the same way as in [3] it should also be possible to relate the two actions. Although our work supports the pure spinor formulation it does not really clarify what constraints should be imposed on $\lambda$. Partly this is a consequence of the fact that we started from the pure-spinor formulation and tried to obtain the $\kappa$-symmetric formulation rather than the other way around. 


\section{Conclusions}

The main results presented in this paper are meant to support the pure spinor formulation of the supermembrane developed by Berkovits. Here we tried to relate the conventional BRST charge in the kappa-symmetric formulation with the postulated "BRST charge" for the pure spinor formulation of the supermembrane and we argue that it is posible to reinstate the reparameterisation constraints in the pure-spinor formulation of the supermembrane by introducing a topological sector and performing a similarity transformation and that the resulting BRST charge would be of conventional type, related to the BRST charge of the $\kappa$-symmetric supermembrane in a formulation where all second class constraints are 'gauge unfixed' to first class constraints.

In our analysis we also encountere a natural candidate for a (non-covariant) supermembrane analogue of the superstring $b$ ghost.

\section{A. Conventions and technical details}

In this appendix we collect our conventions and some technical details. Our conventions are closely related to those of [8], but with some minor differences. Spacetime indices are labeled by capital letters from the middle of the alphabet: $M, N, \ldots=0, \ldots, 9,11$. Spinor indices are labeled by capital letters from the beginning of the alphabet: $A, B, \ldots=1, \ldots, 32$. The gamma matrices $\left(\Gamma^{M}\right)^{A}{ }_{B}$ satisfy the usual algebra: $\left\{\Gamma^{M}, \Gamma^{N}\right\}=\frac{1}{2}\left(\Gamma^{M} \Gamma^{N}+\Gamma^{N} \Gamma^{M}\right)=\eta^{M N}$. We worked with the mostly plus metric $\eta^{M N}=\operatorname{diag}(-+\ldots+)$. Indices can be lowered using the charge-conjugation matrix $C_{A B}=-C_{B A}$ via $\left(\Gamma^{M}\right)_{A B}=C_{A D}\left(\Gamma^{M}\right)^{D}{ }_{B}$ and raisedwith the inverse of this matrix. We do not write $C_{A B}$ explicitly as the position of the indices should always be clear from the context. Also, we do not write the spinor indices explicitly in fully contracted expressions. $\Gamma^{M_{1} \cdots M_{p}}$ is symmetric for $p=1,2,5$ and antisymmetric for $p=0,3,4\left(p=0\right.$ corresponds to $\left.C_{A B}\right)$; these form a basis for the bispinor $\Psi_{A} \Upsilon_{B}$ as

$$
\begin{aligned}
\Psi_{A} \Upsilon_{B}= & \frac{1}{32}\left[(\Psi \Upsilon) C_{A B}+\left(\Psi \Gamma^{S_{1}} \Upsilon\right)\left(\Gamma_{S_{1}}\right)_{A B}-\frac{1}{2 !}\left(\Psi \Gamma^{S_{1} S_{2}} \Upsilon\right)\left(\Gamma_{S_{1} S_{2}}\right)_{A B}-\right. \\
& -\frac{1}{3 !}\left(\Psi \Gamma^{S_{1} S_{2} S_{3}} \Upsilon\right)\left(\Gamma_{S_{1} S_{2} S_{3}}\right)_{A B}+\frac{1}{4 !}\left(\Psi \Gamma^{S_{1} S_{2} S_{3} S_{4}} \Upsilon\right)\left(\Gamma_{S_{1} S_{2} S_{3} S_{4}}\right)_{A B}+ \\
& \left.+\frac{1}{5 !}\left(\Psi \Gamma^{S_{1} S_{2} S_{3} S_{4} S_{5}} \Upsilon\right)\left(\Gamma_{S_{1} S_{2} S_{3} S_{4} S_{5}}\right)_{A B}\right] .
\end{aligned}
$$

Any antisymmetric bispinor $f^{[A B]}$ in eleven dimensions can be decomposed into a scalar, a 3-form and a 4-form

$$
f^{[A B]}=C^{A B} f+\left(\Gamma_{M N P}\right)^{A B} f^{M N P}+\left(\Gamma_{M N P Q}\right)^{A B} f^{M N P Q},
$$

and any symmetric bispinor $g^{(A B)}$ into a 1-form, a 2-form and a 5-form

$$
g^{(A B)}=\left(\Gamma_{M}\right)^{A B} f^{M}+\left(\Gamma_{M N}\right)^{A B} f^{M N}+\left(\Gamma_{M N P Q R}\right)^{A B} f^{M N P Q R} .
$$

We sometimes find it useful to decompose our expressions into a (non-covariant) $\mathrm{U}(5)$ basis. Alternative decompositions are $\mathrm{SO}(8)$ and $\mathrm{SO}(9)$. Under $\mathrm{SO}(11) \rightarrow \mathrm{U}(5)$ a vector decomposes as 
$V^{M} \rightarrow\left(v^{a}, v_{a}, v^{11}\right)$ where

$$
v_{a}=\frac{V^{a}+i V^{a+5}}{2}, \quad v^{a}=\frac{V^{a}-i V^{a+5}}{2}, \quad v^{11}=V^{11} .
$$

$>$ From which it follows that e.g. $U_{M} V^{M}=2 u_{a} v^{a}+2 u^{a} v_{a}+u^{11} v^{11}$. Tensors are decomposed in a similar way.

A spinor $\Psi^{A}$ splits as $\left(\psi_{\alpha}, \psi_{\dot{\alpha}}\right)$ and then further as $\psi_{\alpha} \rightarrow\left(\psi^{+}, \psi^{a}, \psi_{[a b]}\right)$ and $\psi_{\dot{\alpha}} \rightarrow\left(\psi^{-}, \psi_{a}, \psi^{[a b]}\right)$ where $a, b=1, \ldots, 5$.

In the $U(5)$ basis the gamma matrices can be chosen as

$$
\begin{array}{ll}
\left(\gamma^{1}\right)_{A}{ }^{B}=\frac{\sigma^{1}+i \sigma^{2}}{2} \otimes \mathbb{1} \otimes \mathbb{1} \otimes \mathbb{1} \otimes \mathbb{1}, & \left(\gamma^{2}\right)_{A}{ }^{B}=\sigma^{3} \otimes \frac{\sigma^{1}+i \sigma^{2}}{2} \otimes \mathbb{1} \otimes \mathbb{1} \otimes \mathbb{1}, \\
\left(\gamma^{3}\right)_{A}{ }^{B}=\sigma^{3} \otimes \sigma^{3} \otimes \frac{\sigma^{1}+i \sigma^{2}}{2} \otimes \mathbb{1} \otimes \mathbb{1}, & \left(\gamma^{4}\right)_{A}{ }^{B}=\sigma^{3} \otimes \sigma^{3} \otimes \sigma^{3} \otimes \frac{\sigma^{1}+i \sigma^{2}}{2} \otimes \mathbb{1}, \\
\left(\gamma^{5}\right)_{A}{ }^{B}=\sigma^{3} \otimes \sigma^{3} \otimes \sigma^{3} \otimes \sigma^{3} \otimes \frac{\sigma^{1}+i \sigma^{2}}{2}, & C_{A B}=i \sigma^{2} \otimes \sigma^{1} \otimes i \sigma^{2} \otimes \sigma^{1} \otimes i \sigma^{2}, \\
\left(\gamma_{1}\right)_{A}{ }^{B}=\frac{\sigma^{1}-i \sigma^{2}}{2} \otimes \mathbb{1} \otimes \mathbb{1} \otimes \mathbb{1} \otimes \mathbb{1}, & \left(\gamma_{2}\right)_{A}{ }^{B}=\sigma^{3} \otimes \frac{\sigma^{1}-i \sigma^{2}}{2} \otimes \mathbb{1} \otimes \mathbb{1} \otimes \mathbb{1}, \\
\left(\gamma_{3}\right)_{A}{ }^{B}=\sigma^{3} \otimes \sigma^{3} \otimes \frac{\sigma^{1}-i \sigma^{2}}{2} \otimes \mathbb{1} \otimes \mathbb{1}, & \left(\gamma_{4}\right)_{A}{ }^{B}=\sigma^{3} \otimes \sigma^{3} \otimes \sigma^{3} \otimes \frac{\sigma^{1}-i \sigma^{2}}{2} \otimes \mathbb{1}, \\
\left(\gamma_{5}\right)_{A}{ }^{B}=\sigma^{3} \otimes \sigma^{3} \otimes \sigma^{3} \otimes \sigma^{3} \otimes \frac{\sigma^{1}-i \sigma^{2}}{2}, & \left(\Gamma^{11}\right)_{A}{ }^{B}=\sigma^{3} \otimes \sigma^{3} \otimes \sigma^{3} \otimes \sigma^{3} \otimes \sigma^{3},
\end{array}
$$

where $\sigma^{1,2,3}$ are the usual Pauli matrices

$$
\sigma^{1}=\left(\begin{array}{ll}
0 & 1 \\
1 & 0
\end{array}\right), \quad \sigma^{2}=\left(\begin{array}{cc}
0 & -i \\
i & 0
\end{array}\right), \quad \sigma^{3}=\left(\begin{array}{cc}
1 & 0 \\
0 & -1
\end{array}\right) .
$$

Using the $U(5)$ decomposition, we can write formulæ for $\lambda \xi, \lambda \Gamma^{M} \xi$ and $\lambda \Gamma^{M N} \xi$ in the following form

$$
\begin{aligned}
\lambda \xi= & \lambda^{+} \xi^{-}-\lambda^{-} \xi^{+}+\lambda^{a} \xi_{a}-\lambda_{a} \xi^{a}+\frac{1}{2} \lambda_{a b} \xi^{a b}-\frac{1}{2} \lambda^{a b} \xi_{a b} \\
\lambda \Gamma^{11} \xi= & \lambda^{+} \xi^{-}+\lambda^{-} \xi^{+}+\lambda^{a} \xi_{a}+\lambda_{a} \xi^{a}+\frac{1}{2} \lambda_{a b} \xi^{a b}+\frac{1}{2} \lambda^{a b} \xi_{a b} \\
\lambda \gamma^{a} \xi= & -\lambda^{+} \xi^{a}-\lambda^{a} \xi^{+}+\lambda_{b} \xi^{a b}+\lambda^{a b} \xi_{b}+\frac{1}{4} \varepsilon^{a b c d e} \lambda_{b c} \xi_{d e} \\
\lambda \gamma_{a} \xi= & \lambda^{-} \xi_{a}+\lambda_{a} \xi^{-}-\lambda^{b} \xi_{a b}-\lambda_{a b} \xi^{b}-\frac{1}{4} \varepsilon_{a b c d e} \lambda^{b c} \xi^{d e} \\
\lambda \gamma^{a} \Gamma^{11} \xi= & \lambda^{+} \xi^{a}+\lambda^{a} \xi^{+}+\lambda_{b} \xi^{a b}+\lambda^{a b} \xi_{b}-\frac{1}{4} \varepsilon^{a b c d e} \lambda_{b c} \xi_{d e} \\
\lambda \gamma_{a} \Gamma^{11} \xi= & \lambda^{-} \xi_{a}+\lambda_{a} \xi^{-}+\lambda^{b} \xi_{a b}+\lambda_{a b} \xi^{b}-\frac{1}{4} \varepsilon_{a b c d e} \lambda^{b c} \xi^{d e} \\
\lambda \gamma^{a b} \xi= & -\lambda^{+} \xi^{a b}-\lambda^{a b} \xi^{+}-\frac{1}{2} \varepsilon^{a b c d e}\left(\lambda_{c d} \xi_{e}+\lambda_{e} \xi_{c d}\right) \\
\lambda \gamma_{a b} \xi= & \lambda^{-} \xi_{a b}+\lambda_{a b} \xi^{-}+\frac{1}{2} \varepsilon_{a b c d e}\left(\lambda^{c d} \xi^{e}+\lambda^{e} \xi^{c d}\right) \\
\lambda \gamma_{b}^{a} \xi= & \lambda^{a} \xi_{b}+\lambda_{b} \xi^{a}+\lambda_{b c} \xi^{c a}+\lambda^{a c} \xi_{c b} \\
& +\frac{1}{2} \delta_{b}^{a}\left(\lambda^{+} \xi^{-}+\lambda^{-} \xi^{+}-\lambda^{c} \xi_{c}-\lambda_{c} \xi^{c}+\frac{1}{2} \lambda_{c d} \xi^{c d}+\frac{1}{2} \lambda^{c d} \xi_{c d}\right)
\end{aligned}
$$

The pure spinor condition $\lambda \Gamma^{M} \lambda=0$ writen in the $\mathrm{U}(5)$ representation becomes

$$
\begin{aligned}
-\lambda^{+} \lambda^{a}+\lambda^{a b} \lambda_{b}+\frac{1}{8} \varepsilon^{a b c d e} \lambda_{b c} \lambda_{d e} & =0, \\
\lambda^{-} \lambda_{a}-\lambda_{a b} \lambda^{b}-\frac{1}{8} \varepsilon_{a b c d e} \lambda^{b c} \xi^{d e} & =0, \\
\lambda^{+} \lambda^{-}+\lambda^{a} \lambda_{a}+\frac{1}{4} \lambda_{a b} \lambda^{a b} & =0 .
\end{aligned}
$$


$>$ From (A.8) si (A.9) we can eliminate the dependent variabiles $\lambda^{a}$ and $\lambda_{a}$ and so we obtain that $\lambda^{A}$ has $32-10=22$ independent components. But (A.10) is invariant to $\left(\lambda_{\alpha}, \lambda_{\dot{\alpha}}\right) \rightarrow\left(\rho \lambda_{\alpha}, \rho^{-1} \lambda_{\dot{\alpha}}\right)$. This means, $\lambda^{A}$ has 23 degrees of freedom $\left(\lambda^{+}, \lambda^{-}, \lambda^{a b}, \lambda_{a b}, \rho\right)$.

Sometimes we find it useful to decompose further into U(4). In this case we write

$$
\begin{aligned}
\lambda^{a} & \rightarrow\left(\lambda^{a^{\prime}}, \kappa^{+}\right), & & \lambda_{a} \rightarrow\left(\lambda_{a^{\prime}}, \kappa^{-}\right), \\
\lambda^{a b} & \rightarrow\left(\lambda^{a^{\prime} b^{\prime}}, \kappa^{a^{\prime}}\right), & \lambda_{a b} & \rightarrow\left(\lambda_{a^{\prime} b^{\prime}}, \kappa_{a^{\prime}}\right),
\end{aligned}
$$

and similarly for $\xi^{A}$.

The corresponding formulæ for $\lambda \xi, \lambda \Gamma^{M} \xi$ and $\lambda \Gamma^{M N} \xi$ in the $\mathrm{U}(4)$ basis can be obtained from (A.7) by inserting the expressions (A.11) (we will not write the result explicitly). To simplify the notation, below we drop the prime and use $a, b=1, \ldots, 4$.

In the $\mathrm{U}(4)$ basis one can write explicit solutions to the $\lambda \Gamma^{M} \lambda=0$ constraint, for example

$$
\begin{aligned}
\lambda^{a}= & \frac{1}{\lambda^{+} \kappa^{e} \lambda_{e}}\left[\kappa^{-} \kappa^{a} \kappa^{b} \lambda_{b}+\lambda^{a b} \lambda_{b} \kappa^{c} \lambda_{c}-\lambda^{+} \lambda^{a b} \lambda_{b c} \kappa^{c}-\frac{1}{8} \kappa^{a} \varepsilon^{b c d e} \lambda_{b c} \lambda_{d e}\right. \\
& \left.-\frac{1}{2} \kappa^{a} \lambda^{+} \lambda^{b c} \lambda_{b c}+\frac{1}{8} \lambda^{a b} \lambda_{b} \varepsilon^{c d e f} \lambda_{c d} \lambda_{e f}-\frac{1}{2} \lambda^{+} \lambda^{-} \varepsilon^{a b c d} \lambda_{b} \lambda_{c d}\right], \\
\kappa_{a}= & -\frac{1}{\kappa^{e} \lambda_{e}}\left[\lambda^{-} \lambda^{+} \lambda_{a}-\kappa^{-} \lambda_{a b} \kappa^{b}-\lambda_{a b} \lambda^{b c} \lambda_{c}-\frac{1}{2} \lambda^{+} \lambda_{a b c d} \kappa^{b} \lambda^{c d}\right], \\
\kappa^{+}= & \frac{\varepsilon^{a b c d} \lambda_{a b} \lambda_{c d}-8 \kappa^{a} \lambda_{a}}{8 \lambda^{+}},
\end{aligned}
$$

which shows that $\lambda$ has 23 independent components.

It is also possible to write down explicit solutions to the $\lambda \Gamma^{M} \lambda=0=\lambda \Gamma^{M N} \lambda$ constraints, as

$$
\begin{array}{r}
\lambda^{a}=\frac{1}{2 \lambda^{+}} \varepsilon^{a b c d} \kappa_{b} \lambda_{c d}, \quad \kappa^{+}=\frac{1}{8 \lambda^{+}} \varepsilon^{a b c d} \lambda_{a b} \lambda_{c d}, \\
\lambda_{a}=\frac{1}{2 \lambda^{-}} \varepsilon_{a b c d} \kappa^{b} \lambda^{c d}, \quad \kappa^{-}=\frac{1}{8 \lambda^{-}} \varepsilon_{a b c d} \lambda^{a b} \lambda^{c d}, \\
\lambda^{a b}=2 \frac{\kappa^{[a} \varepsilon^{b] c d e} \kappa_{c} \lambda_{d e}+2 \lambda^{+} \lambda^{-} \varepsilon^{a b c d} \lambda_{c d}}{\varepsilon^{g h k} \lambda_{f g} \lambda_{h k}},
\end{array}
$$

which shows that such a $\lambda$ has 16 independent components.

We need to mention that we found the GAMMA package [11] very useful in handling the large amount of gamma matrix algebras needed in our calculations. 


\section{References}

[1] M. Băbălîc, N. Wyllard, Towards relating the kappa-symmetric and pure spinor versions of the supermembrane, JHEP 04 (2000) 018 [hep-th/ 0808 . 3691].

[2] N. Berkovits, Super-Poincaré covariant quantization of the superstring, JHEP 04 (2000) 018 [hep-th/0001035].

[3] N. Berkovits, Explaining the pure spinor formalism for the superstring, JHEP 01 (2008) 065 [hep-th/0712.0324].

[4] E. Bergshoeff, E. Sezgin, P. K. Townsend, Supermembranes and eleven-dimensional supergravity, Phys. Lett. B 189 (1987) 75.

[5] N. Berkovits, Towards covariant quantization of the supermembrane, JHEP 09 (2002) 051 [hep-th/0201151].

[6] L. Anguelova, P. A. Grassi, P. Vanhove, Covariant one-loop amplitudes in D = 11, Nucl. Phys. B 702 (2004) 269 [hep-th/0408171].

[7] E. Bergshoeff, E. Sezgin, Y. Tanii, Hamiltonian formulation of the supermembrane, Nucl. Phys. B 298 (1988) 187.

[8] Y. Aisaka, Y. Kazama, Towards pure spinor type covariant description of supermembrane: An approach from the double spinor formalism, JHEP 05 (2006) 041 [hep-th / 0603004 ].

[9] Y. Aisaka and Y. Kazama, Origin of pure spinor superstring, JHEP 05 (2005) 046 [hep-th/0502208].

[10] I. Oda, M. Tonin, On the b-antighost in the pure spinor quantization of superstrings [hep-th/0409052].

[11] U. Gran, GAMMA: A Mathematica package for performing Gamma-matrix algebra and Fierz transformations in arbitrary dimensions, hep-th/0105086v2. 